\title{
The Usability Evaluation Concerning Emotional Responses of Users on Learning Management System
}

\author{
Nattaporn Phongphaew ${ }^{+}$and Arisara Jiamsanguanwong \\ Department of Industrial Engineering, Faculty of Engineering, Chulalongkorn University, Thailand
}

\begin{abstract}
Usability evaluation itself was not guarantee user satisfaction of learning management system (LMS), while an emotional response of user is worth being considered. The purpose of present study was to examine the relationship of each usability attributes and emotional responses of user in order to understand the relationships of these two evaluation methods. Usability testing was conduct on myCourseVille with 5 usability attributes; learnability, efficiency, effectiveness, memorability, and satisfaction. Along with emotional responses for each tasks. Results revealed that user emotional responses were significant correlated with learnability and satisfaction attributes. Implication from this study was for LMS software developer to improve user interface in term of usability and user emotional responses while using system.
\end{abstract}

Keywords: learning management system, usability testing, emotional responses.

\section{Introduction}

Information technology (IT) has seen as a key factor to improve efficiency of work, reduce complexity, and increase work performance. Especially in education area, Learning Management System (LMS) is the IT that helps people manage their learning activities such disseminate teaching materials, and manage information and communication [1]. While, there were some LMS that were not successfully adopted because of its difficulties with implementation, complexity to use, and dissatisfaction reported from users [2].

To avoid improper use and user dissatisfaction from the LMS interface design, usability evaluation was one of the design methods to identify human-computer interface issues and user experiences [3, 4]. Moreover, the LMS that passed the process of usability evaluation would increase their probability to be fully adopted to use in university [5]. Usability was defined as an extent to which a product can be used by specified users to achieve specified goals with effectiveness, efficiency, and satisfaction in a specified context of use [6].

On the other hand, a number of studies suggested that emotional or affective responses while using a product were crucial consideration of product adoption or non-adoption by end-user [7]. It influenced user satisfaction [7], [8]. However, some usability products were rarely designed concerning customer emotional responses. Thus, there were not success in the marketplace [9].

Usability evaluation alone was not guarantee user satisfaction of system [10]. Some previous studies had introduced the usability evaluation as objective measurement along with user's emotional responses as subjective measurement [4]. To the best of our knowledge, there was no study focusing on how each usability attributes relate to emotional responses of user in the context of LMS. Thus, the purpose of present study was to examine the relationship of each usability attributes and emotional responses of user in order to understand the relationships of those two evaluation methods. Implication from this study was a valuable information for LMS software developer to improve user interface in term of usability and user emotional responses while using system.

\footnotetext{
Corresponding author. Tel.: + 6681-572-4443; fax: +66-2251-3969.

E-mail address: Nattaporn.Ph@student.chula.ac.th
} 


\section{Theory and Research Hypotheses}

\subsection{Usability attributes}

Usability was evaluated on five attributes according to Nielsen [11] and ISO 9241-11 [6]; learnability, efficiency, memorability, effectiveness, and satisfaction. Meanwhile, the Error attribute from Nielsen [11] was considered as a combination factor with the effectiveness attribute from the ISO 9241-11 [6] because the Error attribute was defined as error rate that similarly to the accuracy and completeness in the effectiveness attribute. Moreover, the ability to solve the error in the Error attribute was measured along with learnability attribute.

According to the study of Frøkjær and Hertzum [12] claimed that the subjective measurement as satisfaction and the objective measurements as efficiency and effectiveness were weak correlations and not well understood in context of complex task [12]. Thus, the associations between the usability attributes were investigated as:

H1: Learnability has a positive direct effect on satisfaction.

$\mathrm{H} 2$ : Memorability has a positive satisfaction.

H3: Efficiency has a positive influence on satisfaction.

H4: Effectiveness has a positive influence on satisfaction.

\subsection{Usability and emotional responses}

Emotional response was an important factor when user considered adoption of new product [7]. It also influenced on user's satisfaction and perception of usability [7, 13]. Also, some researcher suggested that usability with multi-functionality itself was not enough anymore, technology should provide remarkable affective experience to the user [8]. The emotional response is defined as personal experience that spontaneously and unconsciously occurs such as anger, sadness and enjoy when was stimulated by environment [14].

Tzvetanova, Tang, and Justice [15] found that higher positive valance will occur with the greater adaptive interface. Similar to Thüring and Mahlke [13] that the well-designed system with good usability provided positive valance on user, while the ill-designed system with poor usability provided negative valance on user. However, it remained unclear of how each usability attributes influence on user emotional responses as positive-negative valence while using LMS. Thus, we hypothesized the following regarding usability and emotional responses:

H5: Learnability has a positive influence on emotion responses.

H6: Effectiveness has a positive influence on emotion responses.

H7: Emotion response has a positive influence on satisfaction.

\section{Methodology}

\subsection{Participants}

Thirty undergraduate and graduate students from science based faculty participated in this study for their credits from the usability-related class. Seventeen are male. Their age ranged from 20 to 24 years $(M=21.16$, $S D=1.02$ ). Experiments were conducted with one participant at a time. All participants had more than 5 year internet-used experience. All participants were novice user of LMS used in this study.

\subsection{Stimulus material}

\subsection{1. myCourseVille}

MyCourseVille version 1.9.3 was used as an LMS in present study. It was implemented for supporting learning activities by Engineering faculty, Chulalongkorn University [16]. Main functions of myCourseVille for students were download course materials, submit assignment, search the course schedule, search the statistics of class attendance, gather the working group, and search the midterm score.

\subsubsection{Affective pictures stimuli}

International Affective Picture System (IAPS) [17] was used to manipulate participants' emotional state. 
IAPS is the set of pictures that had the standard rating valence score from 1: unpleasant, to 9: pleasant. Twenties pictures were selected with the standard valence score for male $(M=4.83, S D=0.25)$ and female $(M=4.89, S D=0.35)$. Mutilation and erotic imagery was excluded.

\subsection{Apparatus}

The private closed room was set with the facilities. The Window-PC was installed the local browser for setting myCourseVille. Screen recording software and microphone were set up for recording activities on the screen and voice. Two video cameras were set up to record facial expression and movement during the experiment. The 65"television was set up in front of the table for introduce the objective of testing and explain the testing instruction.

\subsection{Measurement}

\subsubsection{Usability attributes}

The usability attributes consisted of learnability, efficiency, memorability, effectiveness, and satisfaction which were described following:

- Learnability - the completion time each tasks when use the system for the first time. A lower of completing time means ease in learning the system [11].

- Efficiency - the completion time each tasks when familiar with the system. A shorter spending time indicated better efficiency [18].

- Memorability - the completion time each tasks when not using the system for a periods. A shorter completing time mean better memorability [11].

- Effectiveness - the task success ratio (TSR) is the product of completion ratio multiplies with accuracy ratio. Completion ratio is the actual number of page change divided by expected number of page change. Accuracy ratio is the actual number of click divided by expected number of click [18].

- Satisfaction - after scenario questionnaire at the end of each task and The Post-Study System Usability Questionnaire at the end of each round [19].

\subsubsection{Tasks}

The participants were asked to complete the tasks scenario in myCourseVille in order to evaluate usability of interface design. Seven tasks were set; register to the course, download a course material, submit assignment, search the course schedule, search the statistics of class attendance, gather the working group, and search the midterm score. Average standard time and standard working paths of tasks were determined from three expert users (Cronbach's alpha 0.993).

\subsubsection{After-scenario questionnaire}

After-Scenario Questionnaire (ASQ) [19] was used to measure user satisfaction when participants completed each task by asking individuals opinion to the statements with 7-point Likert scales, "Strongly disagree" for 1, "Strongly agree" for 7. The ASQ consists of 3 question-items regarding to ease of completion task, completion time, and adequacy of support information (on-line help, messages, and documentation). A higher average score means greater user satisfaction.

\subsubsection{Post-study system usability questionnaire}

Post-Study System Usability Questionnaire (PSSUQ) [19] was used for measuring user satisfaction with system usability at the end of each round. This includes 19 items questionnaires asking individuals opinion to the statements with 7-point Likert scales, "Strongly disagree" for 1, "Strongly agree" for 7. Average score was used for the user satisfaction score.

\subsubsection{Self-assessment manikin}

Self-Assessment Manikin (SAM) [20] was used in present study to access participants' affective states in term of valence dimension of emotion after use the system. SAM is a self-report measurement of people's affective states with 9-point scales represented by the facial graphic from unhappy (1) to happy (9). Participants were asked to choose the number that best described their emotion at that moment. 


\subsection{Test procedure}

Prior to beginning the experiment, participants were given a brief explanation of the purpose of the research and were asked to read and sign a consent form. Participants were then asked to complete demographic questionnaire and were informed that the purpose of viewing the following images was to manipulate their emotions. Twenties pictures of neutral content from IAPS [17] were each presented for $6 \mathrm{~s}$, with a 3-s interval between pictures. After viewing the 20 pictures, participants were asked to complete the SAM questionnaire [20] to rate their present emotional valence.

Participants were then presented an overview instruction of the experiment and informed that the experiment consists of 3 rounds. This was followed by the first round. Participants were asked to complete each of the 7 tasks scenarios without the instruction or help information supporting in order to measure the learnability and effectiveness. After finished each task, participants were asked to complete SAM [20] and ASQ questionnaires [19]. At the end of the first round, participants were asked to complete PSSUQ questionnaire [19] for overall satisfaction.

The second round, participants were instructed and practiced to use system for all 7 tasks and they were then asked to complete each of 7 tasks scenarios again in order to measure efficiency. Participants were asked to complete ASQ questionnaire each tasks and also PSSUQ at the end of the second round. Then, participants were informed to have the third round after 7 days. During those 7 days, please avoided from practice or using this system.

The third round, participants were asked to complete all 7 tasks in order to measure memorability. They were again asked to complete ASQ questionnaire at the end of each tasks and also PSSUQ at the end of the third round before dismissed.

\section{Results}

Four participants were excluded from the analyses due to outlier from distribution. All participants could complete all tasks. Mean and standard deviations of time, task success ratio (TSR), and satisfaction scores (ASQ, PSSUQ) each round and standard time for all tasks were presented on Table 1. Effectiveness of system was illustrated by the task success ratio that demonstrated the complexity of use.

\subsection{Usability attributes}

The Pearson correlation coefficient between learnability, efficiency, effectiveness, memorability and satisfaction were analyzed as shown in Table 2. There were positive significant correlation between efficiency, effectiveness, and memorability with learnability and also positive significant correlation between memorability with efficiency.

The results showed that satisfaction of user was significant influenced from learnability of the system by showing negative significant correlation between ASQ scores with learnability. This confirmed hypothesis 1 . The more people used time to complete the tasks, the less satisfaction they reported. Also, there was a negative significant correlation between ASQ scores with effectiveness. This supported hypothesis 4 . The more people committed errors during tasks, the less satisfaction they reported. Moreover there were positive significant correlations between user satisfactions in each round (ASQ, PSSUQ).

\subsection{Usability attributes and emotion responses}

The Pearson correlation coefficient between usability attributes and emotional responses were analyzed as shown in Table 2. There were positive correlations between user satisfaction (ASQ, PSSUQ) and emotional response. This confirmed hypothesis 7. Participants reported more satisfaction when they rated more positive valence of emotional response. Moreover, the results also revealed that participants' emotional response have negative significant influenced from learnability of the system. This supported our hypothesis 5. The shorter the time used to complete tasks for the first time, the more positive valence of user emotional response.

\section{Discussion and Conclusion}

The purpose of present study was to examine the relationship of each usability attributes and emotional 
response of users. Usability evaluation method along with user emotional response during the experiment was conducted. The results revealed that the learnability and effectiveness of system had significant influence on user satisfaction. A longer duration time and errors would reduce user satisfaction. LMS software developer should aware of these issues in order to improve user satisfaction. Furthermore, there were positive significant correlations between user satisfactions in each round (ASQ, PSSUQ), this implied that user satisfaction from the first round have an influence on their satisfaction rating on second and third round.

The significant correlation between learnability and emotional responses inferred that participant's emotional responses were evoked with the ease of use of the system, especially for the first time experience. Moreover, participant's emotional responses were correlated with both of each task satisfaction and overall satisfaction for all rounds. This supported that user's satisfaction were influenced by the emotion of first time interaction with the system. Clearly that the first time using experience was the majority factor that influence to the user's satisfaction and emotional response.

For future works, the impact of affective response should be investigated with experienced user. This may provide more clearly impact of emotional response and their using behavior with the system. Limitation of this study was regarding to the scope of this study. Participants were limited to university students from science-based faculty. It is expected to have some differences regarding to their faculty. For example, the people who study in the field of art may provide some different results of how their emotional responses influence their behaviors. Thus, it would be worth investigating with broader range of people from various countries, different generations, and people with different backgrounds in future study.

Table 1. Mean and standard deviation of time, task success ratio (TSR), and satisfaction scores (ASQ, PSSUQ) each round and standard time for all tasks

\begin{tabular}{|c|c|c|c|c|c|c|c|c|}
\hline \multirow{2}{*}{ Task } & \multicolumn{4}{|c|}{ Average time on task (SD) } & \multirow{2}{*}{$\begin{array}{c}\text { TSR (SD) } \\
\text { Round } 1\end{array}$} & \multicolumn{3}{|c|}{ ASQ (SD) } \\
\hline & Round 1 & Round 2 & Round 3 & Standard & & Round 1 & Round 2 & Round 3 \\
\hline 1 & $41.93(19.57)$ & $18.13(3.90)$ & $21.67 \quad(4.70)$ & 18.00 & $2.64(3.74)$ & $5.08(1.02)$ & $5.78(0.97)$ & $5.82(0.91)$ \\
\hline 2 & $24.43(33.36)$ & $9.93(2.36)$ & 10.80 & 10.00 & $5.43(20.08)$ & $4.99(1.02)$ & $5.73(0.98)$ & $5.88(0.94)$ \\
\hline 3 & $109.23(47.32)$ & $38.43(5.58)$ & $48.30(10.28)$ & 36.67 & $2.81 \quad(2.83)$ & $4.68(1.06)$ & $5.63(0.99)$ & $5.71(1.00)$ \\
\hline 4 & $16.73(7.16)$ & $8.90(1.75)$ & $10.43 \quad(3.33)$ & 8.33 & $2.09(1.66)$ & $5.22(1.03)$ & $5.84(1.06)$ & $5.87(0.99)$ \\
\hline 5 & $66.13(48.14)$ & $12.17(3.37)$ & 14.43 (2.99) & 11.67 & $9.88(14.03)$ & $4.56(1.35)$ & $5.67(0.97)$ & $5.71(0.97)$ \\
\hline 6 & $83.73(35.54)$ & $33.17(7.35)$ & $38.57(10.28)$ & 32.33 & $2.94 \quad(2.41)$ & $4.89(0.99)$ & $5.71(0.97)$ & $5.90(0.88)$ \\
\hline 7 & $12.70(6.39)$ & $6.27(1.01)$ & $(1.11)$ & 5.33 & $3.27 \quad(4.03)$ & $5.35(1.02)$ & $5.93(1.00)$ & $6.08(0.94)$ \\
\hline $\begin{array}{l}\text { PSSUQ } \\
\text { (SD) }\end{array}$ & - & - & - & - & - & $4.91(0.92)$ & $5.40(1.01)$ & $5.42(1.09)$ \\
\hline
\end{tabular}

Table 2. Pearson Correlation Coefficient between Usability Attributes and Emotional Responses

\begin{tabular}{|c|c|c|c|c|c|c|c|c|c|c|c|}
\hline Variable & 1 & 2 & 3 & 4 & 5 & 6 & 7 & 8 & 9 & 10 & 11 \\
\hline 1. Learnability & 1 & & & & & & & & & & \\
\hline 2. Efficiency & $.761^{* *}$ & 1 & & & & & & & & & \\
\hline 3. Memorability & $.769^{* *}$ & $.913^{* *}$ & 1 & & & & & & & & \\
\hline 4. Effectiveness & $.363^{* *}$ & -.048 & -.038 & 1 & & & & & & & \\
\hline 5. ASQ.R1 ${ }^{\mathrm{a}}$ & $-.283^{* *}$ & -.075 & -.087 & $-.193^{*}$ & 1 & & & & & & \\
\hline 6. ASQ.R $2^{\mathrm{b}}$ & -.093 & -.027 & .007 & .040 & $.647^{* *}$ & 1 & & & & & \\
\hline 7. ASQ.R3 $^{\mathrm{c}}$ & -.121 & -.017 & -.025 & -.071 & $.612^{* * *}$ & $.839^{* * *}$ & 1 & & & & \\
\hline 8. Emotion $\mathrm{R} 1^{\mathrm{a}}$ & $-.178^{*}$ & -.082 & -.036 & -.104 & $.675^{* *}$ & $.584^{* *}$ & $.548^{* *}$ & 1 & & & \\
\hline 9. PSSUQ.R1 ${ }^{\mathrm{a}}$ & -.044 & .021 & .056 & -.002 & $.641^{* *}$ & $.745^{* *}$ & $.632^{* *}$ & $.596^{* *}$ & 1 & & \\
\hline 10. PSSUQ.R2 ${ }^{\mathrm{b}}$ & -.013 & .048 & .040 & -.005 & $.541^{* * *}$ & $.788^{* * *}$ & $.807^{* * *}$ & $.636^{* *}$ & $.781^{* * *}$ & 1 & \\
\hline 11. PSSUQ.R3 ${ }^{\mathrm{c}}$ & -.012 & .052 & .056 & -.010 & $.505^{* *}$ & $.792^{* *}$ & $.823^{* * *}$ & $.583^{* *}$ & $.671^{* *}$ & $.923^{* *}$ & 1 \\
\hline
\end{tabular}

${ }^{\mathrm{a}}$ score of $1^{\text {st }}$ round testing, ${ }^{\mathrm{b}}$ score of $2^{\text {nd }}$ round testing, ${ }^{\mathrm{c}}$ score of $3^{\text {rd }}$ round testing.

$* * p<0.01, * p<0.05$

\section{References}

[1] Coates, H., R. James, and G. Baldwin, A critical examination of the effects of learning management systems on university teaching and learning. Tertiary Education and Management, 2005. 11: p. 19-36.

[2] van der Linden, J. and C. van de Leemput, Observatory of students' uses of computer-based tools. Psychologie 
Française, 2015. 60(2): p. 145-157.

[3] Buzhardt, J., et al., Usability testing of the classwide peer tutoring-learning management system. Journal of Special Education Technology, 2005. 20(1): p. 19-29.

[4] Ulbricht, V.R., et al., The emotion component on usability testing human computer interface of an inclusive learning management system, in International Limousine, Charter \& Tour Conference. 2014: Las Vegas, USA. p. 334-345.

[5] Ramakrisnan, P., et al., Evaluation of user interface design for leaning management system (LMS): Investigating student's eye tracking pattern and experiences. Procedia - Social and Behavioral Sciences, 2012. 67: p. $527-537$.

[6] ISO 9241-11, Ergonomic requirements for office work with visual display terminals (VDTs), Part 11: Guidance on usability. 1998.

[7] Partala, T. and T. Saari, Understanding the most influential user experiences in successful and unsuccessful technology adoptions. Computers in Human Behavior, 2015. 53: p. 381-395.

[8] Umemuro, H., Affective Technology, Affective Management, towards Affective Society, in Human-Computer Interaction. Ambient, Ubiquitous and Intelligent Interaction: 13th International Conference, HCI International 2009, San Diego, CA, USA, July 19-24, 2009, Proceedings, Part III, J.A. Jacko, Editor. 2009, Springer Berlin Heidelberg: Berlin, Heidelberg. p. 683-692.

[9] Khalid, H.M., Customer Emotional Needs in Product Design. Concurrent Engineering, 2006. 14(3): p. $197-206$.

[10] Croll, J., Testing for usability is not enough: Why clinician acceptance of health information systems is also crucial for successful implementation. International Federation for Information Processing, 2010. 335: p. 49-60.

[11] Nielsen, J., Usability engineering. 1993, San Francisco: Morgan Kaufman.

[12] Frøkjær, E., M. Hertzum, and K. Hornb, Measuring usability: are effectiveness, efficiency, and satisfaction really correlated?, in Proceedings of the SIGCHI conference on Human Factors in Computing Systems. 2000, ACM: The Hague, The Netherlands. p. 345-352.

[13] Thüring, M. and S. Mahlke, Usability, aesthetics and emotions in human-technology interaction. International Journal of Psychology, 2007. 42(4): p. 253-264.

[14] Coleman, A.D., in Dictionary of psychology. 2009, Oxford University Press: New York.

[15] Tzvetanova, S., M.-X. Tang, and L. Justice, Emotional Web Usability Evaluation, in Human-Computer Interaction. HCI Applications and Services: 12th International Conference, HCI International 2007, Beijing, China, July 22 27, 2007, Proceedings, Part IV, J.A. Jacko, Editor. 2007, Springer Berlin Heidelberg: Berlin, Heidelberg. p. 10391046.

[16] Trisahaveerakul, P., Business plan of courseville sustainable development on higher education in Thailand, in Faculty of Commerce and Accountancy. 2014, Chulalongkorn University.

[17] Lang, P.J., M.M. Bradley, and B.N. Cuthbert, International affective picture system (IAPS): Technical manual and affective ratings NIMH Center for the Study of Emotion and Attention, 1997: p. 39-58.

[18] Lin, C., Exploring the relationship between technology acceptance model and usability test. Information Technology and Management, 2013. 14(3): p. 243-255.

[19] Lewis, J.R., IBM computer usability satisfaction questionnaires: Psychometric evaluation and instructions for use. 1993, IBM Corporation. p. 1-34.

[20] Bradley, M.M. and P.J. Lang, Measuring emotion: The self-assessment manikin and the semantic differential. Journal of Behavior Therapy and Experimental Psychiatry, 1994. 25(1): p. 49-59. 